\title{
Rotationally resolved fluorescence-dip and ion-dip spectra of single rovibronic states of benzene
}

\author{
Th. Weber, E. Riedle, and H. J. Neusser \\ Institut für Physikalische und Theoretische Chemie, Technische Universität München, Lichtenbergstrasse 4, \\ D-8046 Garching, Federal Republic of Germany
}

Received March 5, 1990; accepted May 10, 1990

\begin{abstract}
We report fluorescence-dip as well as ion-dip spectra of single rovibronic one-photon states of benzene with a linewidth as narrow as $0.14 \mathrm{~cm}^{-1}$. The selective excitation of the rovibronic states was achieved through the combination of a frequency-doubled pulsed amplified cw dye laser $\left(\Delta \nu_{\mathrm{Uv}} \approx 100 \mathrm{MHz}\right)$ and a collimated molecular beam. The detailed analysis of the dip spectra shows that the observed spectral features correspond to single rovibronic transitions if suitable states are excited. From the spectra, precise harmonic frequencies and anharmonic constants for the $S_{0}$ state are determined. A hitherto unknown Darling-Dennison resonance of the overtones of $\nu_{1}$ with the $5_{2}$ state is found.
\end{abstract}

\section{INTRODUCTION}

In molecular spectroscopy the many rotational lines are assigned according to a given zero-order Hamiltonian to the various quantum numbers that are associated with rotation, and the spectroscopic parameters are then extracted. In many cases deviations from line positions predicted by the Hamiltonian are seen as perturbations in the spectrum, and analyses of these can sometimes yield higher-order corrections to the Hamiltonian. These corrections or couplings between the different zero-order states of the molecule are also responsible for the redistribution of the vibrational or electronic energy of the molecule and give rise to the dynamics observed in timeresolved experiments. The interesting instances of the dynamics in the intermediate case or at the statistical limit leads to a completely perturbed spectrum, and a detailed analysis of the spectrum is nearly impossible. This is because the couplings between the zero-order states lead to a mixture of the wave functions, and therefore many more lines are observed in the spectrum than would be expected from considerations of regular selection rules. To identify the various lines in the spectrum according to the quantum numbers and to eliminate the many contributions from sequence congestion additional information are the purposes of the experiment.

Such information could come from optical doubleresonance schemes. In a $V$-type (or labeling) experiment a selected ground state would be marked by saturation of an assigned rovibronic transition with one laser, and a second laser will detect only those transitions that originate from this known ground state. A simplified spectrum of the excited electronic state $\left(S_{1}\right)$ results; this spectrum contains only coupled states without inhomogeneous sequences. In a $\Lambda$-type (or stimulated-emission pumping) experiment a selected excited state of the molecule is prepared by the first laser pulse, and the excited molecules are monitored by either fluorescence or resonance-enhanced ionization detection. If the frequency of the second laser pulse matches the frequency of a possible transition from the excited state to the electronic ground state, a decrease in the signal is observed. The resulting fluorescence-dip or ion-dip spectra contain only transitions from a single excited state and thus have a greatly simplified structure. Analyses of these dip spectra can then render information on the energy levels and couplings in the $S_{0}$ state.

Dip spectra have been reported for a number of molecules in the past few years. For small polyatomics such as formaldehyde, ${ }^{1,2}$ acetylene, ${ }^{2,3}$ or glyoxal ${ }^{4}$ the selective excitation of single rotational states was possible with commercially available dye lasers, but for larger polyatomics such as $p$-difluorobenzene, ${ }^{5}$ trans-stilbene, ${ }^{6}$ or benzene ${ }^{7}$ only vibrational states could be resolved because of the limited resolving power of the excitation laser. Particularly for these larger systems, the simultaneous simplification of double-resonance spectroscopy and rotational resolution were badly needed, since such large polyatomics possess a highly complicated structure of levels, owing to the many vibrational degrees of freedom and the large number of rotational states populated even under jet conditions.

In a recent study we were able to show that the use of a pulsed amplified cw laser instead of a commercial dye laser permits the selective excitation of single rovibronic states even for a large system such as benzene..$^{8-10}$ In these investigations and in others that were performed with Doppler-free two-photon excitation, ${ }^{11}$ many couplings in the $S_{1}$ state could be analyzed. The ability to selectively excite rovibronic states is now used in the present study to record rotationally resolved dip spectra of single states of benzene. Both fluorescence detection and ion detection are used, and the merits of the two methods are compared. It is found that fairly low spectral resolution of the dip laser is sufficient to resolve the rotational structure of the dip spectra completely. The spectra give a greatly simplified picture of the highly complex rovibrational level structure at an intermediate vibrational excess energy in the $S_{0}$ state. The analysis of the spectra yields the harmonic frequencies of the observed vibra- 
tional modes, their anharmonic constants, and the nature and magnitude of some hitherto unobservable couplings.

\section{EXPERIMENTS}

The experimental setup used in the present investigations is similar to those used previously to record rotationally resolved one-photon fluorescence excitation spectra of benzene $e^{9,12}$ and two-color resonance-enhanced ionization spectra of benzene ${ }^{12}$ and benzene-Ar. ${ }^{10}$ The lastmentioned setup is shown in Fig. 1. Only the features that are particularly relevant to the present study will be described here.

The ability to selectively excite single rovibronic states of benzene, $\mathrm{C}_{6} \mathrm{H}_{6}$, is obtained through the combination of a frequency-doubled pulsed amplified single-mode cw dye laser $\left(\Delta \nu_{\mathrm{UV}} \approx 100 \mathrm{MHz}\right.$, Ref. 9) with a collimated molecular beam of benzene seeded in Ar., ${ }^{9,10}$ The rotational temperature in the beam is $\sim 10 \mathrm{~K}^{9}$ After excitation of the benzene molecules to a rotational level in either the $6^{1}$ vibronic state $(\lambda \approx 258.9 \mathrm{~nm})$ or the $6^{1} 1^{1}$ state $(\lambda \approx 252.9 \mathrm{~nm})$, stimulated emission to rotational levels in excited vibrational levels of $S_{0}$ is induced by means of the frequency-doubled output of a second dye laser (Lambda Physik, FL 2002 E) operated with Coumarin 307 or 153 dye. The frequency width of this laser (in the UV) is $\sim 0.4 \mathrm{~cm}^{-1}$ with only the grating used in the oscillator or $0.10 \mathrm{~cm}^{-1}$ with the intracavity étalon inserted. When the second laser is scanned, we observe a signal from any excited molecules whose frequencies do not match a possible transition frequency, and a reduced signal is obtained for close resonance. In this way dip spectra are recorded that map the structure of the levels in the $S_{0}$ state that can be accessed in a one-photon transition from the originally excited rovibronic state.

The two laser beams are combined collinearly by means of an uncoated quartz plate placed at an angle of $45^{\circ}$. In this way $10 \%$ of the excitation (pump) laser output is overlapped with $90 \%$ of the dip (dump) laser output. The small amount of light from the pump laser is sufficient to saturate the excitation transition partially. ${ }^{9}$ The directions of linear polarization of the two lasers were chosen to be parallel. The size of each beam at the region of interaction with the molecular beam is adjusted separately with a telescope that consists of two lenses, and the beams are carefully overlapped.

So that we could record fluorescence-dip spectra, the pulsed amplifier system for the pump laser was pumped by the output of an EMG 1003 (Lambda Physik) XeCl excimer laser. The size of the UV beam $(\approx 20 \mu \mathrm{J}$ per pulse) at the molecular beam was adjusted to a diameter of $1.8 \mathrm{~mm}$, and this resulted in an intensity of $1.0 \times$

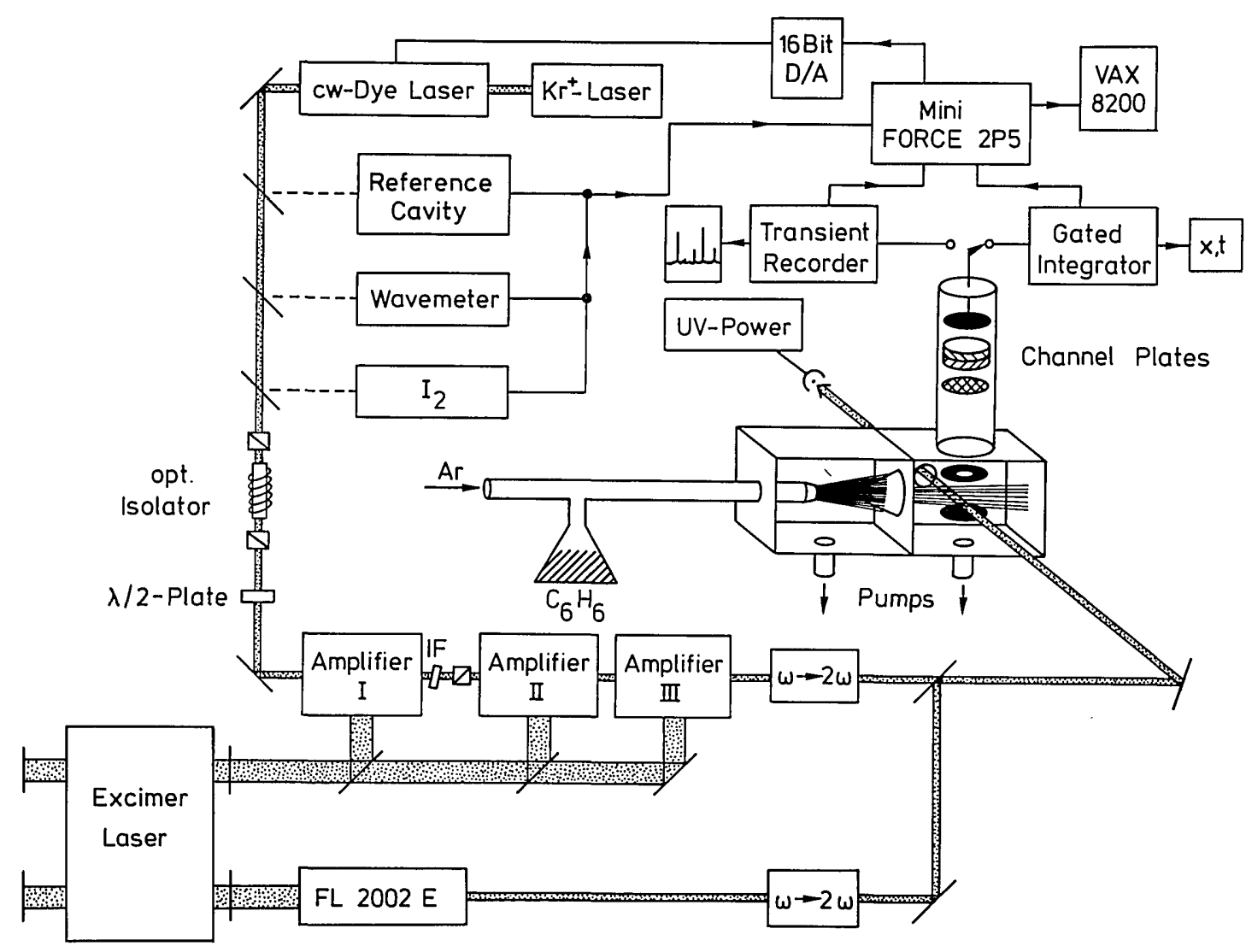

Fig. 1. Experimental setup for recording of rotationally resolved ion-dip spectra of single rovibronic states of benzene (from Ref. 10). A spectral resolution of the excitation (pump) laser of $100 \mathrm{MHz}$ is obtained by pulsed amplification of a single-mode cw dye laser and consequent frequency doubling. The light of the second frequency-doubled dye laser (dump laser) either ionizes the excited molecules or induces the stimulated emission to the ground electronic state. The frequency of the first laser is kept fixed in order to record dip spectra at the desired rovibronic transition, and the second laser is scanned. If, instead, the frequency of the second laser is kept fixed and the first laser is scanned, a resonance-enhanced two-photon ionization spectrum of the excitation band is obtained. ${ }^{10,12}$ 
$10^{5} \mathrm{~W} / \mathrm{cm}^{2}$ for the pulse length of 10 nsec. The dump laser beam was unfocused (with a slightly larger diameter than the pump beam) at a pulse length of $10 \mathrm{nsec}$ and an intensity of $3 \times 10^{5} \mathrm{~W} / \mathrm{cm}^{2}$. The delay between the pump and the dump pulse could be freely chosen, since the pump laser was pumped by an independent excimer laser (one of the two discharge tubes of a model EMG 150). The fluorescence emitted from the electronically excited benzene molecules was detected in a time gate beginning $20 \mathrm{nsec}$ after the dump pulse. To avoid any spurious signal from stray laser light, we installed baffles at the entrance side of the molecular beam chamber. ${ }^{9}$

To permit us to record ion-dip spectra, the two laser systems were pumped by the two discharge tubes of the EMG 150 laser, and the delay was fixed in this way to 11 nsec. $^{12}$ The pump beam was focused to an area of $\sim 0.1 \mathrm{~mm}^{2}$ at the molecular beam. In order to avoid complete saturation of the excitation transition, the beam was attenuated to yield an intensity of $6 \times 10^{5} \mathrm{~W} / \mathrm{cm}^{2}$ in the interaction region. The dump laser was only slightly focused (to an $\sim 3-\mathrm{mm}^{2}$ area), and an intensity of $4 \times$ $10^{6} \mathrm{~W} / \mathrm{cm}^{2}$ resulted. In addition to being used to induce stimulated emission, the dump laser was used to ionize part of the excited molecules, and the resulting ions were detected. ${ }^{10,12}$ This setup is identical to the one used to record resonance-enhanced ionization of benzene ${ }^{12}$ or benzene-Ar ${ }^{10}$ except that in these previous experiments the frequency of the pump laser was scanned and the frequency of the second (dump) laser was fixed to a value for which no transition to vibrationally excited states of $S_{0}$ occurs. In the present experiments, the frequency of the pump laser is held fixed at the desired transition and the dump laser is purposely scanned over a region in which stimulated emission is expected to occur.

The rotational structure of both the $6_{0}^{1}$ and the $6_{0}^{1} 1_{0}^{1}$ band were analyzed previously, ${ }^{9}$ and therefore the energies of all the rotational states in the vibronic states $6^{1}$ and $6^{1} 1^{1}$ are known to a precision of better than $0.01 \mathrm{~cm}^{-1}$. To calibrate the frequency of the dump laser, we use the well-known absorption spectrum of $\mathrm{I}_{2} \cdot{ }^{13}$ In this way the term values of all states that are accessed by stimulated emission can be determined to a precision of $\sim 0.05 \mathrm{~cm}^{-1}$.

\section{RESULTS}

Fluorescence-dip and ion-dip spectra of selected rovibronic states of benzene were recorded. They are presented in Subsections 3.A.1 and 3.A.2, respectively; the influence of the dump-laser linewidth and intensity on the ion-dip spectra will be discussed. In Subsection 3.A.3 the merits of the two types of detection are compared. In Subsection 3.B.1 the spectroscopic background that is needed for interpretation of the observed rotational structure will be reviewed. Next, ion-dip spectra of various rovibronic states will be evaluated, to yield precise spectroscopic information on some of the vibrational states accessible from the $6^{1} 1^{1}$ vibronic state. These are the degenerate $6_{2}$ state (Subsection 3.B.2) and the nondegenerate $1_{2}$ and $1_{3}$ states (Subsection 3.B.3).

\section{A. Characteristics of Dip Spectra}

1. Fluorescence-Dip Spectra

In the lower part of Fig. 2, a 1-cm ${ }^{-1}$ part of the $R$ branch of the rotationally resolved fluorescence excitation spec-
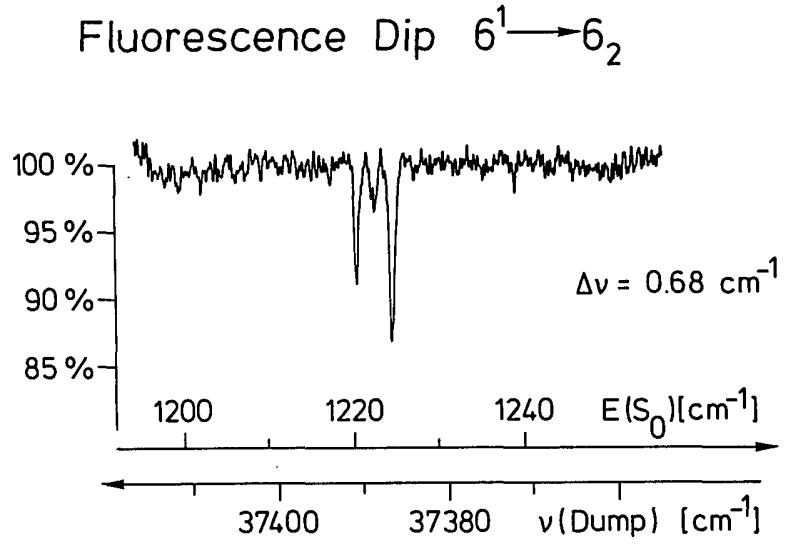

Excitation Spectrum $6_{0}^{1}$

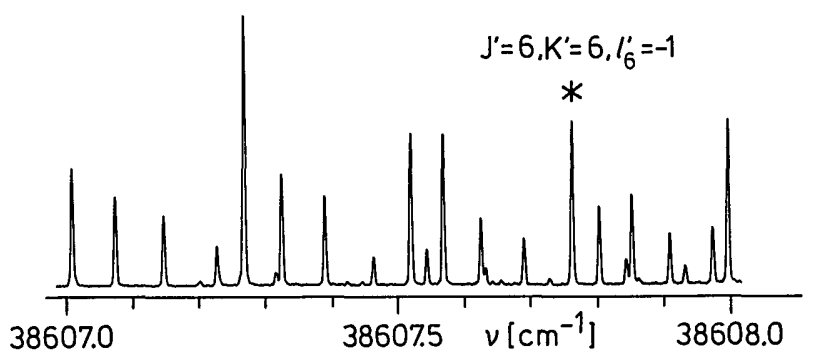

Fig. 2. (Top) Fluorescence-dip spectrum after selective excitation of the $J^{\prime}=6, K^{\prime}=6, l_{6}^{\prime}=-1$ rotational state of the $6^{1}$ vibronic state of benzene, $\mathrm{C}_{6} \mathrm{H}_{6}$. The spectral range covered corresponds to the various rotational states accessible in the $\mathbf{6}_{2}$ vibrational state. Both the frequency scale of the dump laser and the energy (sum of vibrational and rotational energy) of the $S_{0}$ levels accessed are given. (Bottom) For reference, part of the fluorescence excitation spectrum of the $6_{0}^{1}$ band $^{9}$ is shown. The rovibronic transition to the $J^{\prime}=6, K^{\prime}=6, l_{6}^{\prime}=-1$ state used for excitation is marked by an asterisk.

trum of the $6_{0}^{1}$ band of benzene, ${ }^{9}$ located $\sim 45 \mathrm{GHz}$ to the blue from the rotationless origin, is reproduced. It consists of well-separated lines that correspond to single rovibronic transitions. Selected rovibronic states of the molecule can be accessed by means of these transitions. In a previous publication ${ }^{9}$ we noted that the pulse-to-pulse fluctuations of the fluorescence signal are less than $10 \%$ in our experiment thanks to the homogeneous and stable frequency distribution of the pulsed amplified $\mathrm{cw}$ laser used for the excitation of the molecules. In addition, these fluctuations are highly correlated with the UV power, as can be confirmed by variation of the laser power. Owing to the partial saturation of the transitions at the intensities used, ${ }^{9}$ the power dependence of the signal is not linear, but it can be represented by part of a parabolic curve for the range of interest. We are therefore able to normalize the observed signal for each laser pulse to a fitted parabola and to reduce the rms value of the fluctuation, after averaging over 10 laser pulses, to less than $1 \%$ without the use of a reference configuration. ${ }^{2,4}$ This can be seen in the upper part of Fig. 2, where the fluorescence signal that originates from excitation of the $J^{\prime}=6, K^{\prime}=$ $6, l_{6}^{\prime}=-1$ rotational state (marked with an asterisk in the lower part of Fig. 2) is shown. During this 10-min recording of the fluorescence signal, the dump laser was scanned from $37355 \mathrm{~cm}^{-1}$ to $37417 \mathrm{~cm}^{-1}$. Near 
$37390 \mathrm{~cm}^{-1}$ three dips in the fluorescence signal of as much as $13 \%$ are seen, which correspond to stimulated emission down to rotational levels of the $6_{2}$ vibrational state. The width of the dips is $0.68 \mathrm{~cm}^{-1}$. Since the energy of the initially excited rovibronic state is precisely known ${ }^{9}$ and the frequency of the dump laser is well calibrated (see Section 2), we can directly determine the term values (sum of vibrational and rotational energy) of all the ground states that are accessed by the stimulated emission. The corresponding scale of $S_{0}$ energies is shown immediately below the experimental curve in the upper part of Fig. 2.

\section{Ion-Dip Spectra}

In Fig. 3 ion-dip spectra obtained after excitation of the $J^{\prime}=4, K^{\prime}=4, l_{6}^{\prime}=-1$ rotational level of the $6^{1} 1^{1}$ vibronic level of benzene are shown for three different conditions of the dump (and ionization) laser. Again the spectral range for transitions to the $6_{2}$ state are shown. The spectrum at the bottom of Fig. 3 was recorded with only the grating used as the selective element in the dump laser oscillator, and a $0.73-\mathrm{cm}^{-1}$ width of the dips results, which is quite similar to the width observed in the fluorescence-dip spectrum of Fig. 2. If the intracavity étalon is used to narrow the laser linewidth, the width of the dips decreases to $0.25 \mathrm{~cm}^{-1}$ (see the top trace in

$$
\text { ION DIP } 6^{1} 1^{1}\left(J^{\prime}=4, K^{\prime}=4, l_{6}^{\prime}=-1\right) \longrightarrow 6_{2}
$$
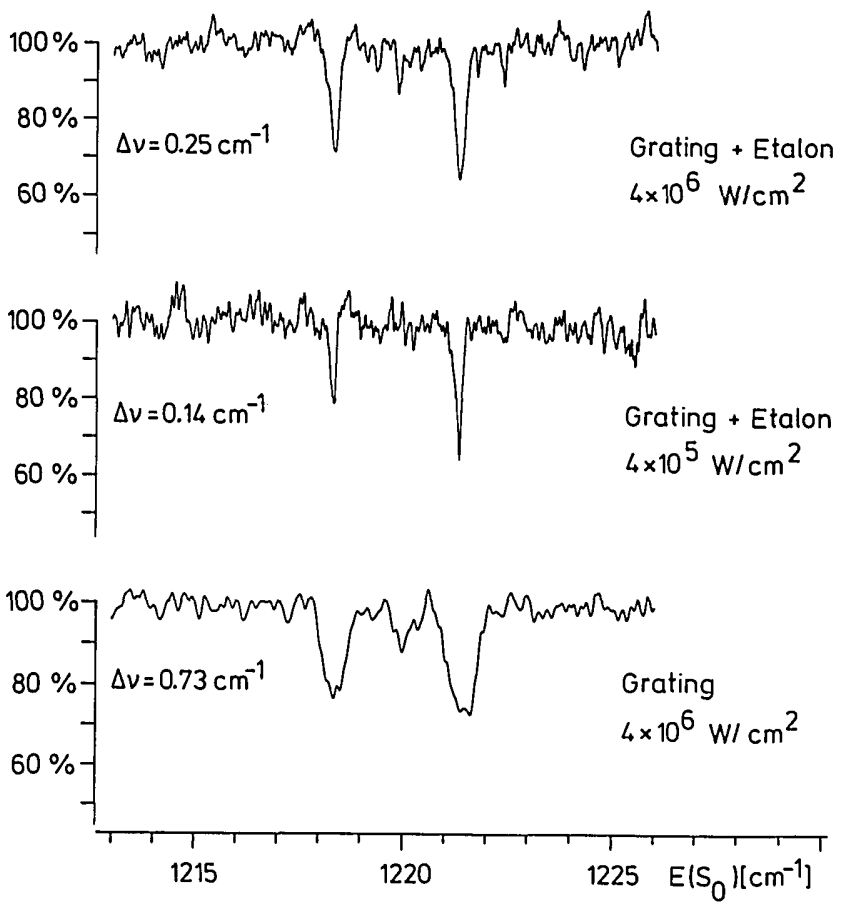

Fig. 3. Ion-dip spectra after excitation of the $J^{\prime}=4, K^{\prime}=4$, $l_{6}^{\prime}=-1$ rotational state of the $6^{1} 1^{1}$ vibronic state. For the recording shown in the bottom trace, the dump laser was operated with only the grating as selective element. For the upper two traces, an additional étalon in the oscillator was used to narrow the laser bandwidth. The relatively high intensity used for the recording of the top trace was decreased for the middle recording in order to avoid power broadening of the spectrum; this alteration results in a narrower dip width.
Fig. 3). This is still significantly greater than the UV linewidth of the dump laser of $0.10 \mathrm{~cm}^{-1}$ and can be understood as saturation broadening of the stimulated transition. If we reduce the intensity of the dump laser by a factor of 10, the observed width of the dips does indeed decrease to $0.14 \mathrm{~cm}^{-1}$ (see the middle trace of Fig. 3). A further decrease of the intensity did not decrease the width of the dips by much but caused a strong decrease in the depth of the dips. As a matter of fact, in the spectrum shown in the middle trace of Fig. 3 the dip at $E\left(S_{0}\right)=$ $1220 \mathrm{~cm}^{-1}$ is already hardly recognizable.

The observed dip depth of more than $30 \%$ in the ion-dip spectra needs some comment. If the excited rovibronic state and the electronic ground-state level that correspond to a given dip are considered to be a two-level system, a maximum dip depth of $50 \%$ is expected. We must, however, keep in mind that the frequency distribution of the dump laser consists of a few longitudinal modes that are spaced by $\sim 5$ times their individual width and fluctuate in frequency from pulse to pulse. The inherent width of each dump transition is determined in our experiment by the small residual Doppler broadening ${ }^{9}$ of less than $50 \mathrm{MHz}$ and is therefore negligible. Hence for the average of many laser pulses a dip depth of less than $50 \% / 5=10 \%$ should be observable. We believe that, owing solely to saturation broadening, the dump laser can be held in resonance for every laser shot, and dips of significantly more than $10 \%$ can be observed. The postulation of saturation broadening at the intensities used is in good agreement with recent observations for benzene by Esherick et al. ${ }^{14}$ Inherently, then, the width of each dip must be larger than the linewidth of the dump laser. Only if weak dips could be detected could a width close to the laser linewidth be achieved. For ionization detection, however, we are not able to normalize the signal to the laser power because we would have to account for fluctuations in both lasers and not just for fluctuations of the pump laser. Therefore a much larger fluctuation of the signal is observed for ionization detection (compare Fig. 3) than was seen in fluorescence detection (see Fig. 2 and the discussion above).

\section{Comparison of Fluorescence-Dip and Ion-Dip Spectra}

Suzuki et al. ${ }^{6}$ pointed out that, under the conditions of the energy levels that concern us and for the frequency of the ionization threshold at which both fluorescence dips and ion dips can be observed, ion dips are experimentally more easily observed because the measured signal originates from the same region of the molecular sample in which the stimulated emission takes place, regardless of the quality of the overlap of the two beams. In addition, in ion-dip spectroscopy stray light from either one of the two lasers is not a problem, while in fluorescence detection it is often a severe problem. We can emphatically confirm these ideas. Despite numerous efforts (a change in the relative size of the two beams, variation of the delay from the optimum value of $\sim 20 \mathrm{nsec}$ ), no fluorescence dips stronger than the ones shown in Fig. 2 could be observed. Rather, we found it hard to reproduce this level of dip depth, presumably because of day-to-day changes in the exact beam profiles. On the other hand, ion dips of as much as $40 \%$ were easily and routinely observed, as can be seen from Figs. 4 and 5. This depth did depend only on the intensity of the dump laser. As was mentioned above, too low 


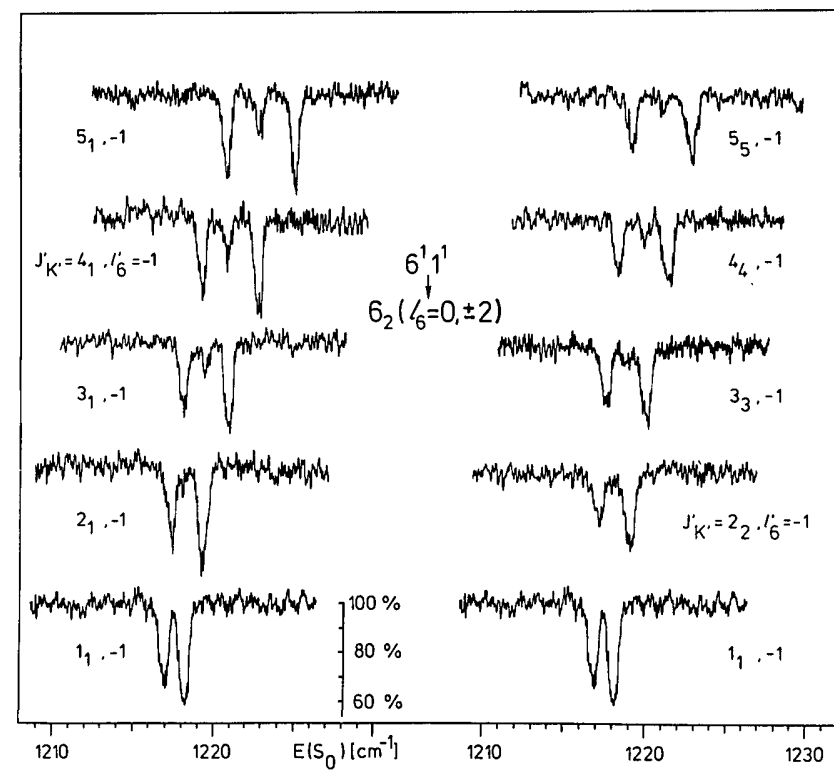

Fig. 4. Ion-dip spectra after selective excitation of $1 \leqq J^{\prime}=$ $K^{\prime} \leqq 5, l_{6}^{\prime}=-1$ and $1 \leqq J^{\prime} \leqq 5, K^{\prime}=1, l_{6}^{\prime}=-1$ rotational states of the $6^{1} 1^{1}$ vibronic state. The spectral range covered corresponds to the energy spread of the various rotational states accessible in the $6_{2}$ vibrational state. For assignment and evaluation of the spectra see text.

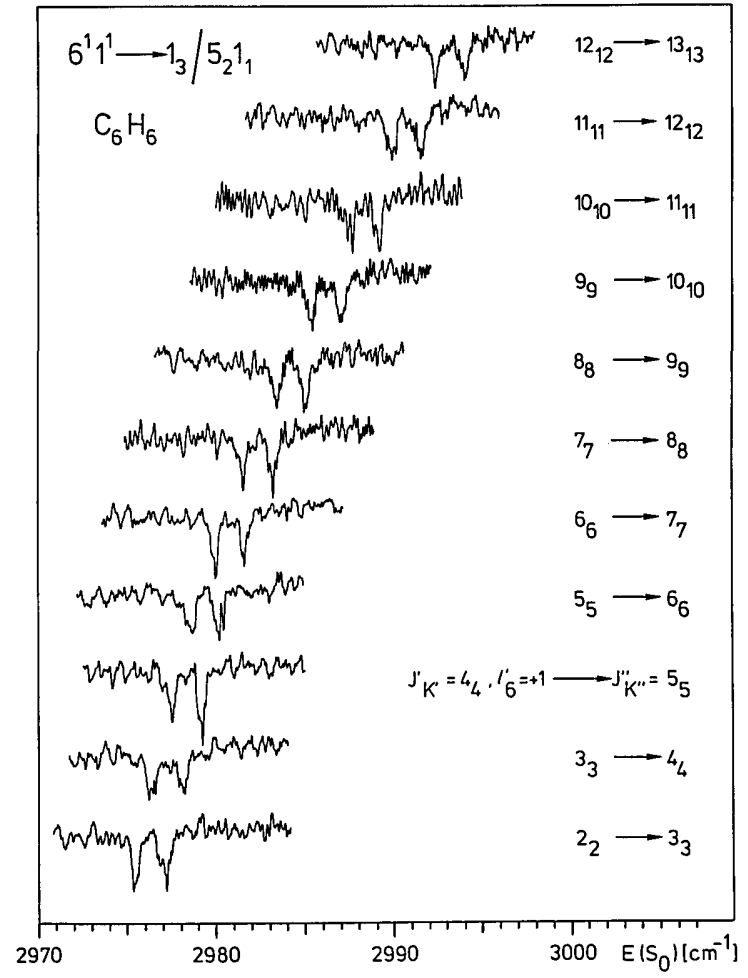

Fig. 5. Ion-dip spectra after selective excitation of $1 \leqq J^{\prime}=$ $K^{\prime} \leqq 12, l_{6}^{\prime}=+1$ rotational states of the $6^{1} 1^{1}$ vibronic state. The spectral range covered corresponds to the energy spread of the rotational states accessible in the $1_{3}$ vibrational state. Note that each spectrum consists of two peaks separated by a constant energy difference. For explanation see text.

an intensity caused a strong decrease of the dip depth, but a further increase of the intensity also resulted in weaker dips. We believe that this result is due to the fact that at high intensities of the dump laser most excited molecules are ionized regardless of possible stimulated emission. In conclusion, ionization detection is by far the easier way to find the frequencies of possible stimulated emission, but the signal-to-noise ratio of the dip spectra (which is given by the ratio of the dip depth and the fluctuation of the total signal) is slightly better in fluorescence detection.

\section{B. Spectroscopic Results}

\section{Spectroscopic Background: Rotational Structure} of Dip Spectra

All the dip spectra presented above display a fine structure that will now be interpreted as the rotational structure. From this analysis it will follow that the individual features correspond to single rovibronic transitions in most cases, and we can therefore determine precise spectroscopic constants from the spectra. Examples will be given in Subsections 3.B.2 and 3.B.3 for the $6_{2}$ and for the $1_{2}$ and $1_{3}$ states, respectively.

The energy $E_{0}(v, l, J, K)$ of a rovibronic level above the zero-point energy of the oblate symmetric top molecule benzene can be conveniently expressed as

$$
E_{0}(v, l, J, K)=G_{0}(v, l)+E_{\text {rot }}(J, K, l) .
$$

$G_{0}(v, l)$ is the vibronic part of the energy (denoted by $\nu_{00}$ in our earlier publications ${ }^{9,10}$ ) and depends on the manifolds of vibrational quantum numbers $v_{i}$ and vibrational angular momentum quantum numbers $l_{i}$ as ${ }^{15,16}$

$$
G_{0}(v, l)=T_{e}+\sum_{i} \omega_{i}{ }^{0}{ }^{0}{ }_{i}+\sum_{i} \sum_{j \geqq i} x_{i j}{ }^{0} v_{i} v_{j}+\sum_{i} \sum_{j \geqq i} g_{i j} l_{i} l_{j} .
$$

$T_{e}$ denotes the energy of electronic state, $\omega_{i}{ }^{0}$ is the harmonic frequency of the $i$ th vibrational mode, and $x_{i j}{ }^{0}$ and $g_{i j}$ are the usual anharmonic constants of the electronic state in question. A splitting of the vibrational angular momentum substates of a multiple degenerate overtone or combination state is given for nonvanishing values of $g_{i j}$.

The rotational energy $E_{\text {rot }}(J, K, l)$ is given by ${ }^{15}$

$$
E_{\mathrm{rot}}(J, K, l)=B_{v} J(J+1)+\left(C_{v}-B_{v}\right) K^{2} \mp 2 C_{v} \zeta_{\mathrm{eff}} K,
$$

with the rotational constants $B_{v}$ and $C_{v}$ of the vibronic state, its effective Coriolis coupling constant $\zeta_{\text {eff }}$, and the rotational quantum numbers $J$ and $K$. The last term in Eq. (1c) describes the splitting of each rotational state in a degenerate vibronic state into the $(+l)$ or and $(-l)$ vibrational angular momentum substates due to Coriolis coupling with the minus or plus sign to be used, respectively. According to Hoy and Mills ${ }^{17}$ a substate is labeled $(+l)$ if

$$
\operatorname{sign}\left[k \sin \left(2 \pi g_{\mathrm{ev}} / n\right)\right]
$$

is positive and $(-l)$ if this quantity is negative. The quantity $k$ in expression (2) is the signed version of the rotational quantum number $K, g_{\mathrm{ev}}$ is the signed version of Hougen's quantum number $G_{\mathrm{ev}}$, which will be described below, and $n$ is the order of the primary axis of symmetry in the molecule. For details the reader is referred to the original paper. ${ }^{17}$ In addition, Hoy and Mills were able to relate the value of $\zeta_{\text {eff }}$ for an arbitrary degenerate vibronic 
state, described by the values of $l_{i}$, to the individual $\zeta_{i}$ constants through the equation

$$
\zeta_{\mathrm{eff}}=\operatorname{sign}\left[\sin \left(2 \pi g_{\mathrm{ev}} / n\right)\right] \sum_{i} l_{i} \zeta_{i} .
$$

The information given so far permits the determination of the energy of any particular rovibronic state of benzene if all the spectroscopic constants and quantum numbers are known. To decide which rovibronic transitions can be observed, we must turn to the selection rules.

Generally, strong vibronic one-photon transitions in benzene are observed for a change by \pm 1 in the vibrational quantum number $v_{6},{ }^{18}$ and these give rise to perpendicular bands. ${ }^{15}$ Accordingly, the rotational selection rules

$$
\Delta J=0, \pm 1, \quad \Delta K= \pm 1
$$

apply. In addition, at least one of the two vibronic states involved is degenerate and is therefore split by Coriolis coupling. To determine which substates will give rise to a transition, symmetry selection rules must be invoked. These were formalized by Hougen ${ }^{19}$ through his quantum number $G$, defined (modulo $n$ ) by

$$
\begin{aligned}
G & =G_{\mathrm{ev}}-K \\
& =G_{e}+(n / 2) \sum_{B} v_{B}+\sum_{j} s_{j} l_{j}-K,
\end{aligned}
$$

where the first summation runs over all nondegenerate vibrational modes of species $B$ and the second over all degenerate ones. For electronic states of $A$ symmetry, $G_{e}=0$, for $B$ states $G_{e}=n / 2$, and for $E_{s}$ states $G_{e}=s ; s_{j}$ is determined by the symmetry $E_{s}$ of the $j$ th degenerate vibrational mode. The symmetry selection rule is then reduced to the simple equation

$$
\Delta G=0 ;
$$

i.e., transitions are allowed if the symmetry of the rovibronic wave function and accordingly the quantum number $G$ are not changed. The relative intensity of a transition is given by the well-known Hönl-London factors. ${ }^{20}$

\section{Dip Spectra in the $6_{2}^{1} 1_{0}^{1}$ Transition Range}

So that we could record dip spectra of individual rotational states $J^{\prime}, K^{\prime}$, and $l_{6}^{\prime}$ of the $6^{1} 1^{1}$ vibronic state, the states were excited by means of the well-resolved rovibronic transitions of the $6{ }_{0}^{1} 1_{0}^{1}$ band. $^{9}$ The dump laser was scanned over the spectral range that corresponds to transitions to the $6_{2}$ state, and for convenience ionization detection was used (see Subsection 3.A.3). The first examples of the resulting ion-dip spectra are shown in Fig. 3, and a larger number of spectra are shown in Fig. 4. These particular spectra were recorded with the grating resolution of the dump laser, but we also recorded spectra with the narrower linewidth that was given by the additional étalon in the oscillator. Generally speaking, the étalon spectra showed better-resolved lines and allowed a better determination of the line frequencies, but there were increased fluctuations in the ion signal. Our present understanding of these fluctuations is that, with the étalon used, only a few longitudinal modes are present in the frequency spectrum of the dump laser and that these give rise to a slowly varying degree of mode beating, which is coupled to a varying efficiency of the frequency doubling. The increase of the number of longitudinal modes on removal of the étalon tends to reduce the effect of the mode beating, and a more stable UV intensity is observed, which in turn leads to a more stable ion signal.

According to the rules summarized in Subsection 3.A.1, the $( \pm l)$ states of the $6^{1} 1^{1}$ state correspond to $l_{6}^{\prime}=\mp 1$ and can be reached by $\Delta K= \pm 1$ transitions. The $6_{2}$ state is threefold degenerate because of the possible values of $l_{6}^{\prime \prime}=$ $0, \pm 2$. Of the degenerate $l_{6}^{\prime \prime}= \pm 2$ pair of substates, the $l_{6}^{\prime \prime}=-2$ state is the $(+l)$ state. To determine which $J^{\prime}$, $K^{\prime}, l_{6}^{\prime}$ states can be connected to $J^{\prime \prime}, K^{\prime \prime}, l_{6}^{\prime \prime}$ states by the dump transition, we calculated the value of $G^{\prime}$ and $G^{\prime \prime}$ for all possible states. The result is summarized in Table 1 , where $G^{\prime \prime}$ is given relative to $G^{\prime}$ for all possible values $l_{6}^{\prime}$, $l_{6}^{\prime \prime}$, and $\Delta K$. It should be noted that Hougen's quantum number $G$ does not depend on $J$, since the symmetry of the rotational wave function is only determined by $K$. Inspection of Table 1 shows that for each pair of $l_{6}^{\prime}$ and $\Delta K$ only one of the three values of $l_{6}^{\prime \prime}$ gives $\Delta G=0$; i.e., with $\Delta J$ of 0 or \pm 1 , a total of six transitions is possible from a given excited rovibronic level.

This number of possible transitions is even further reduced for particular choices of $J^{\prime}$ and $K^{\prime}$. To illustrate this point, the possible transitions from three states are analyzed in more detail in Table 2. In this table the relative intensity HL (Hönl-London factor), the expected energy $E$ (for the values of the constants, see below), and the permitted values of $l_{6}^{\prime \prime}$ determined from Table 1 are given for all six possible transitions. Take, for example, the $J^{\prime}=4, K^{\prime}=4, l_{6}^{\prime}=-1$ vibronic state, i.e., a state with $J^{\prime}=K^{\prime}$. The ${ }^{p} Q(\Delta J=0, \Delta K=-1)$ and the ${ }^{p} R$ $(\Delta J=+1, \Delta K=-1)$ transition $^{21}$ would lead to states with $J^{\prime \prime}<K^{\prime \prime}$, which do not exist because $K^{\prime \prime}$ can at most be equal to $J^{\prime \prime}$. In addition, the Hönl-London factor for the $r P$ transition to the $J^{\prime \prime}=5, K^{\prime \prime}=3$ state is smaller by approximately a factor of 50 than those for the ${ }^{p} P$ and ${ }^{r} R$ transitions. Therefore only three transitions can be seen in the dip spectrum of $J^{\prime}=K^{\prime}, l_{6}^{\prime}=-1$ states. These transitions are the two strong features and the weak middle feature of the spectra in Figs. 2 and 3 and in the righthand side of Fig. 4. In other words, the modest resolution of $0.7 \mathrm{~cm}^{-1}$ in these spectra is already sufficient to resolve the rotational structure completely, because only transitions from a single rovibronic state are observed. Without this double resonance, many rovibronic transitions would be hidden beneath the bandwidth of $0.7 \mathrm{~cm}^{-1}$. For

Table 1. Hougen's Quantum Number $G^{\prime \prime}$

\begin{tabular}{rcccc}
\hline$G^{\prime \prime}$ & & $l_{6}^{\prime \prime}=-2$ & $l_{6}^{\prime \prime}=0$ & $l_{6}^{\prime \prime}=+2$ \\
\hline$K^{\prime \prime}=K^{\prime}+1$ & $l_{6}^{\prime}=-1$ & $G^{\prime}$ & $G^{\prime}-2$ & $G^{\prime}+2$ \\
& $l_{6}^{\prime}=+1$ & $G^{\prime}+2$ & $G^{\prime}$ & $G^{\prime}-2$ \\
$K^{\prime \prime}=K^{\prime}-1$ & $l_{6}^{\prime}=-1$ & $G^{\prime}+2$ & $G^{\prime}$ & $G^{\prime}-2$ \\
& $l_{6}^{\prime}=+1$ & $G^{\prime}-2$ & $G^{\prime}+2$ & $G^{\prime}$ \\
\hline
\end{tabular}

${ }^{a} G$ " for the various rotational states in the $6_{2}$ vibrational state that can be accessed by means of stimulated emission from a given $J^{\prime}, K^{\prime}, l_{6}^{\prime}$ rotational level (with Hougen's quantum number $G^{\prime}$ ) in the $6^{1}$ vibronic state. $G^{\prime \prime}$ does not depend on $J^{\prime \prime}$, but it depends on $K^{\prime \prime}$ and $l_{6}^{\prime \prime}$ as shown. A transition is allowed if $G^{\prime \prime}$ equals $G^{\prime}(\Delta G=0)$. For details see text. 
Table 2. Relative Intensity (HL) and Ground-State Energy $(E)^{a}$

\begin{tabular}{cccrcc}
\hline Rotational State & $J^{\prime \prime}$ & $K^{\prime \prime}$ & $l_{6}^{\prime \prime}$ & HL & $E$ \\
\hline$J^{\prime}=4$ & 5 & 5 & -2 & 2.00 & 1221.32 \\
$K^{\prime}=4$ & 5 & 3 & 0 & 0.04 & 1221.72 \\
$l_{6}^{\prime}=-1$ & 4 & 5 & -2 & \multicolumn{2}{c}{ Not possible } \\
& 4 & 3 & 0 & 0.40 & 1219.83 \\
& 3 & 5 & -2 & \multicolumn{2}{c}{ Not possible } \\
& 3 & 3 & 0 & 1.56 & 1218.31 \\
$J^{\prime}=4$ & 5 & 5 & 0 & 2.00 & 1220.21 \\
$K^{\prime}=4$ & 5 & 3 & +2 & 0.04 & 1221.09 \\
$l_{6}^{\prime}=+1$ & 4 & 5 & 0 & \multicolumn{2}{c}{ Not possible } \\
& 4 & 3 & +2 & 0.40 & 1219.20 \\
& 3 & 5 & 0 & \multicolumn{2}{c}{ Not possible } \\
& 3 & 3 & +2 & 1.56 & 1217.68 \\
$J^{\prime}=4$ & 5 & 2 & -2 & 0.93 & 1222.66 \\
$K^{\prime}=1$ & 5 & 0 & 0 & 0.89 & 1222.58 \\
$l_{6}^{\prime}=-1$ & 4 & 2 & -2 & 0.90 & 1220.76 \\
& 4 & 0 & 0 & \multicolumn{2}{c}{ Not possible } \\
& 3 & 2 & -2 & 0.17 & 1219.24 \\
& 3 & 0 & 0 & 1.11 & 1219.16 \\
\hline
\end{tabular}

${ }^{a} \mathrm{HL}$ (given by the Hönl-London factor) and $E$ (in $\mathrm{cm}^{-1}$ ) for all possible rovibronic transitions $(\Delta J=0, \pm 1, \Delta K= \pm 1)$ from selected rotational states (denoted by $J^{\prime}, K^{\prime}$, and $l_{6}^{\prime}$ ) of the $6^{1} 1^{1}$ vibronic state to rotational states (denoted by $J^{\prime \prime}$ and $K^{\prime \prime}$ ) of the $6_{2}$ vibrational state. For each combination of $J^{\prime \prime}$ and $K^{\prime \prime}$, three substrates with $l_{6}^{\prime \prime}=0, \pm 2$ exist, but according to symmetry selection rules (see text) only one of them can be reached from each excited vibronic state. The value of the quantum number $l_{6}^{\prime \prime}$ for this state is indicated in the table. $J^{\prime \prime}$ would be smaller than $K^{\prime \prime}$ for some values of $J^{\prime}, K^{\prime}, \Delta J$ and $\Delta K$, and the transition is therefore not possible, as is indicated. The transition from the $J^{\prime}=4, K^{\prime}=1, l_{6}^{\prime}=-1$ state to the $J^{\prime \prime}=4, K^{\prime \prime}=0$ state is not possible for nuclear spin symmetry reasons if a $P$ - or $R$-branch transition is used for excitation.

$J^{\prime}=K^{\prime}, l_{6}^{\prime}=+1$ excited states, the situation is analogous except that the permitted values of $l_{6}^{\prime \prime}$ differ.

Table 2 also shows that the strong ${ }^{r} R$ transition to lower $S_{0}$ energy leads to a $l_{6}^{\prime \prime}=+2$ substate, and the strong ${ }^{p} P$ transition to higher $S_{0}$ energy leads to a $l_{6}^{\prime \prime}=0$ substate of the $6_{2}$ vibrational state. We were therefore able to deduce the energy of the rotationless $l_{6}^{\prime \prime}=0$ and $l_{6}^{\prime \prime}= \pm 2$ substates from the observed peak positions of a large number of dip spectra from $J^{\prime}=K^{\prime}, l_{6}^{\prime}=-1$ states according to Eq. (1). Since the difference between $B_{v}$ and $B_{0}$ is known to be small compared with the accuracy of our frequency scale (at least for the low rotational quantum numbers considered), ${ }^{22}$ and $C_{v}$ is known to be equal to $B_{v} / 2$ within sufficient accuracy, ${ }^{9,23} B_{v}$ was set equal to the wellknown value ${ }^{23}$ of $B_{0}$, and $C_{v}$ was set equal to $B_{0} / 2$. According to Eq. (3), $\zeta_{\text {eff }}^{\prime \prime}$ is equal to 0.00 for the $l_{6}^{\prime \prime}=0$ substate, and it was determined from the experimental positions of the $l_{6}^{\prime \prime}=+2$ states to $-1.15(5)$. This value agrees nicely with the value of $\zeta_{6}^{\prime \prime}$ determined previously for the $\nu_{6}$ Raman band ${ }^{24}$; notice that Eq. (3) predicts it to be equal to $-2 \zeta_{6}^{\prime \prime}=-1.150$. The newly determined spectroscopic constants for the $6_{2}$ state are summarized in Table 3 together with those previously determined for the $6_{1}$ state. $^{24}$

With the assignment of the dip spectra of $J^{\prime}=K^{\prime}, l_{6}^{\prime}=$ -1 states performed and the spectroscopic constants determined, all the dip spectra shown in Fig. 4 can be well understood. On both sides of the figure the spectra are arranged according to increasing values of $J^{\prime}$ and there- fore increasing values of $J^{\prime \prime}$. Since the scale used is the $S_{0}$ energy, it is clear that the shift of the dips to higher energy is simply due to the increasing energy of the higher rotational states in the $6_{2}$ vibronic state. The dip spectra map the energy position of the accessible states in a direct way unlike normal optical spectra, for which the ordering of the transition frequencies generally does not agree with the ordering of the ground- or excited-state energies. Inspection of the right-hand column in Table 2 shows that for excitation of $K^{\prime}=1, l_{6}^{\prime}=-1$ states the two strong peaks in the dip spectra shown on the left-hand side of Fig. 4 correspond to two transitions each. For these excited states the resolution of our dump laser is not sufficient to resolve all transitions. Therefore depletion of the excited state can simultaneously proceed to two states, and the dips from the $K^{\prime}=1$ states are somewhat deeper than the ones from the $J^{\prime}=K^{\prime}$ states.

The newly determined values of the rotationless energy of the vibrational angular momentum substrates of the $6_{2}$ state allow us to evaluate the value of the harmonic frequency of $\nu_{6}$ and its anharmonic constants. The constant $\mathrm{g}_{66}$ is directly determined from the splitting of the substates, and for the determination of $\omega_{6}^{0}$ and $x_{66}^{0}$ the frequency ${ }^{24}$ of $\nu_{6}$ is also used. The results are included in Table 3.

\section{Dip Spectra in the $6_{0}^{1} 1 \frac{1}{2}$ and $6_{0}^{1} 11_{3}^{1}$ Transition Ranges}

The analysis of the rotational structure of dip spectra for the $6_{2}$ state is complicated by the fact that this vibrational state is threefold degenerate, and possible transitions to all three substates have to be considered. Stimulated emission to a nondegenerate state, for example the $1_{2}$ or $1_{3}$ state of $a_{1 g}$ symmetry, should therefore display an even simpler rotational structure. To determine which transitions should actually be expected, we notice that the $l_{6}^{\prime \prime}=0$ substate of the $6_{2}$ state is also of $a_{1 g}$ symmetry and conclude that only those rotational transitions from a given excited rovibronic state that lead to this substate are permitted. Inspection of Table 2 shows that from

\section{Table 3. Summary of Selected Spectroscopic Constants for the $S_{\mathbf{0}}$ State of Benzene ${ }^{a}$}

\begin{tabular}{|c|c|c|c|}
\hline State & $G_{0}(v, l)\left(\mathrm{cm}^{-1}\right)$ & $\zeta_{\text {eff }}$ & Ref. \\
\hline $1_{1}$ & $993.071(3)$ & 0 & 25 \\
\hline $\mathbf{1}_{2}$ & $1984.6(3)$ & 0 & This paper \\
\hline $5_{2}$ & $1984.4(3)$ & 0 & This paper \\
\hline $\mathbf{1}_{3}$ & $2974.8(2)$ & 0 & This paper \\
\hline $5_{2} 1_{1}$ & $2974.8(2)$ & 0 & This paper \\
\hline $6_{1}(l= \pm 1)$ & $608.10(1)$ & 0.575 & 24 \\
\hline $6_{2}(l=0)$ & $1216.88(2)$ & 0 & This paper \\
\hline $6_{2}(l= \pm 2)$ & $1216.91(4)$ & $-1.15(5)$ & This paper \\
\hline \multicolumn{2}{|c|}{$\left\langle 1_{2}\left|\tilde{H}_{40}\right| 5_{2}\right\rangle=0.48 \mathrm{~cm}^{-1}$} & \multicolumn{2}{|c|}{$\left\langle 1_{3}\left|\tilde{H}_{40}\right| 5_{2} 1_{1}\right\rangle=0.83 \mathrm{~cm}^{-1}$} \\
\hline \multicolumn{2}{|c|}{$\omega_{1}^{0}=993.79(4) \mathrm{cm}^{-1}$} & \multicolumn{2}{|c|}{$x_{11}^{0}=-0.73(2) \mathrm{cm}^{-1}$} \\
\hline \multicolumn{2}{|c|}{$\omega_{6}^{0}=607.81(4) \mathrm{cm}^{-1}}$, & \multicolumn{2}{|c|}{$x_{66}^{0}=0.32(3) \mathrm{cm}^{-1}$} \\
\hline \multicolumn{2}{|c|}{$g_{66}=0.007(60) \mathrm{cm}^{-1}$} & & \\
\hline
\end{tabular}

${ }^{a}$ Summary of spectroscopic constants of the $S_{0}$ state of benzene relevant to and determined in this work. For nondegenerate vibrational states or the $l=0$ substate of a degenerate state the value of $\zeta_{\text {eff }}$ is 0 by definition; for details and definitions see text. 
a $J^{\prime}=K^{\prime}, l_{6}^{\prime}=+1$ state only the ${ }^{p} P$ transition (to $J^{\prime \prime}=$ $J^{\prime}+1, K^{\prime \prime}=K^{\prime}+1$ ) should be seen; i.e., the dip spectrum to the $1_{2}$ or $1_{3}$ state should consist of one single line.

To test this hypothesis, we recorded a large number of dip spectra for the two overtones of $\nu_{1}$. The spectra for $J^{\prime}=K^{\prime}=2$ to 12 and $l_{6}^{\prime}=+1$ (excited by means of the well resolved $P$-branch lines of the $6{ }_{0}^{1} 1_{0}^{1}$ band ${ }^{9}$ and dip frequency to the $1_{3}$ state are shown in Fig. 5. Each spectrum consists of two lines separated by a constant amount instead of the expected single line. A similar splitting of somewhat smaller but also constant size was found for the $1_{2}$ state. Two main peaks were also seen in the dip spectra for the $6_{2}$ state, but there the spacing caused by the rotational structure increased monotonically with increasing values of $J^{\prime}$. The present splitting cannot therefore be due to a poorly understood rotational structure, but it must be due to a splitting of each $J^{\prime \prime}=K^{\prime \prime}$ rotational state of the $1_{2}$ and $1_{3}$ vibrational state. Such a splitting, which does not show any rotational dependence, can only be caused by anharmonic coupling between the $1_{2}$ state and an unknown state $X$ and between the $1_{3}$ state and the state $X 1_{1}$. The energy of the rotationless states was deduced from the measured peak positions according to Eqs. (1), and standard perturbation theory was used to deduce the unperturbed energies and the coupling matrix elements. The results are summarized in Table 3 together with the values of $\omega_{1}^{0}$ and $x_{11}^{0}$ that were determined from our new values and the previously determined energy of the $1_{1}$ state. $^{25}$

To identify the coupled vibrational state $X$ we calculated all vibrational states of $a_{1 g}$ symmetry in the vicinity of the $1_{2}$ state. We used the $S_{0}$ energies as tabulated by Dang$\mathrm{Nhu}$ and Pliva. ${ }^{22}$ Within a harmonic approximation, only four states were found less than $\pm 25 \mathrm{~cm}^{-1}$ from the measured energy of $1984.5 \mathrm{~cm}^{-1}$. These are the $6_{1} 16_{1} 17_{1}$ state at $1973.1 \mathrm{~cm}^{-1}$, the $9_{1} 16_{2}$ state at $1973.8 \mathrm{~cm}^{-1}$, the $5_{2}$ state at $1980.0 \mathrm{~cm}^{-1}$, and the $1_{2}$ state at $1986.2 \mathrm{~cm}^{-1}$. The energy of the first two states is considerably lower than the observed energy of the state $X$, and interaction could be only through a quintic term. Therefore quartic coupling to the $5_{2}$ state seems to be most likely; i.e., we assign the observed anharmonic resonance to a DarlingDennison resonance between $1_{n}$ and $1_{n-2} 5_{2}$. Darling and Dennison have shown that the matrix element for such an interaction should scale $\mathrm{as}^{26}$

$$
\left\langle v_{1}, v_{5}\left|\tilde{H}_{40}\right| v_{1}-2, v_{5}+2\right\rangle \propto\left[v_{1}\left(v_{1}-1\right)\left(v_{5}+1\right)\left(v_{5}+2\right)\right]^{1 / 2} .
$$

For the definition of the $\tilde{H}_{40}$ operator, see Ref. 27. The ratio between the coupling matrix elements for $v_{1}=2$ and $v_{1}=3$ is therefore expected to be $\sqrt{3}=1.73$, in perfect agreement with the observed ratio of 1.73 (see Table 3). This is additional evidence for the proposed assignment. The only fact that is still a little disturbing is that the energy of the $5_{2}$ state would be considerably higher than $2 \times \nu_{5}$. Reference to the original work of Brodersen and Langseth ${ }^{28}$ tells us, however, that these authors determined the frequency of $\nu_{5}$ from the combination states $5_{1} 16_{1}$ and $5_{1} 17_{1}$ and that they did not correct for possible anharmonicity. On the other hand, they assigned a weak band at $595 \mathrm{~cm}^{-1}$ to the transition $v_{5}=1 \leftarrow v_{16}=1$, and a value of $\nu_{5}$ of $993 \mathrm{~cm}^{-1}$ could be deduced from this assign- ment without any possible influence of anharmonicity. This value would place the measured energy of the $5_{2}$ state slightly below $2 \nu_{5}$ and therefore seems like a better choice.

\section{SUMMARY AND CONCLUSIONS}

Fluorescence-dip and ion-dip spectra from single rovibronic states of benzene were reported and compared. The analysis of spectra that correspond to the vibronic transition $6^{1} 1^{1} \rightarrow 6_{2}$ showed that for suitable choices of $J^{\prime}$, $K^{\prime}$, and $l_{6}^{\prime}$ every feature in the spectra corresponds nearly exclusively to a single rotational transition even at a frequency resolution of the dump laser of only slightly better than $1 \mathrm{~cm}^{-1}$. This is due to the double-resonance character of dip spectroscopy. From the rotationally resolved spectra, precise spectroscopic constants of the threefolddegenerate $6_{2}$ state were determined. The analysis of dip spectra leading to the $1_{2}$ and $1_{3}$ state yielded the hitherto unknown (to our knowledge) result that these states are coupled to the $5_{2}$ and $5_{2} 1_{1}$ state, respectively, through a quartic anharmonic resonance.

We have also tried to access higher overtones of $\nu_{1}$ than are discussed in this work but unfortunately have not yet succeeded in finding dips at the appropriate frequencies. Whether this is solely due to experimental insufficiencies or to the weak Franck-Condon factors and the forbidden character of the $S_{1}-S_{0}$ transition in benzene is the subject of ongoing investigations. Possibly other organic molecules with an allowed electronic transition will be a better choice for future work.

The reported investigations show that rotationally resolved dip spectroscopy of single rovibronic states is no longer limited to small polyatomics. The spectral resolution obtained was well below $1 \mathrm{~cm}^{-1}$ and thus more than 1 order of magnitude better than that obtained, for example, in emission spectroscopy of benzene. ${ }^{15,17,29-31} \mathrm{Dip}$ spectroscopy could therefore become a valuable tool for the precise investigation of high-lying rovibrational states within the $S_{0}$ manifold of large molecules. So far these states could at best be accessed through weak overtone transitions. These states are of particular interest since some indication of local mode behavior was previously found for overtones of $\mathrm{C}-\mathrm{H}$ strength vibrations, ${ }^{32}$ but a precise understanding was obscured by the thermal inhomogeneous broadening that was present in the spectra.

\section{ACKNOWLEDGMENTS}

The authors are indebted to E. W. Schlag for his continuous interest in the progress of this work. They thank M. Knerich for valuable experimental help. Financial support from the Deutsche Forschungsgemeinschaft and the Fonds der Chemischen Industrie is gratefully acknowledged.

\section{REFERENCES AND NOTES}

1. D. E. Reisner, R. W. Field, J. L. Kinsey, and H. L. Dai, J. Chem. Phys. 80, 5968 (1984); H. L. Dai, C. L. Korpa, J. L. Kinsey, and R. W. Field, J. Chem. Phys. 82, 1688 (1985).

2. C. E. Hamilton, J. L. IKinsey, and R. W. Field, Annu. Rev. Phys. Chem. 37, 493 (1986).

3. E. Abramson, R. W. Field, D. Imre, K. K. Innes, and J. L. Kinsey, J. Chem. Phys. 80, 2298 (1984); 83, 453 (1985). 
4. D. Frye, H. T. Liou, and H. L. Dai, Chem. Phys. Lett. 133, 249 (1987); D. Frye, L. Lapierre, and H. L. Dai, J. Chem. Phys. 89, 2609 (1988).

5. W. D. Lawrance and A. E. W. Knight, J. Chem. Phys. 76, 5637 (1982); S. H. Kable, J. W. Thoman, Jr., and A. E. W. Knight, J. Chem. Phys. 88, 4748 (1988)

6. T. Suzuki, N. Mikami, and M. Ito, J. Phys. Chem. 90, 6431 (1986).

7. O. Krätzschmar, H. L. Selzle, and E. W. Schlag, Z. Naturforsch. 43a, 765 (1988).

8. U. Schubert, E. Riedle, and H. J. Neusser, J. Chem. Phys. 84, 5326 (1986).

9. E. Riedle, Th. Knittel, Th. Weber, and H. J. Neusser, J. Chem. Phys. 91, 4555 (1989).

10. Th. Weber, A. von Bargen, E. Riedle, and H. J. Neusser, J. Chem. Phys. 92, 90 (1990)

11. E. Riedle, H. Stepp, and H. J. Neusser, Chem. Phys. Lett. 110, 452 (1984); U. Schubert, E. Riedle, H. J. Neusser, and E. W. Schlag, J. Chem. Phys. 84, 6182 (1986); H. Sieber, E. Riedle, and H. J. Neusser, J. Chem. Phys. 89, 4620 (1989).

12. E. Riedle, Th. Weber, U. Schubert, H. J. Neusser, and E. W. Schlag, J. Chem. Phys. "Back to the roots of 'channel three': rotationally resolved spectra of the $6_{0}^{1} 1_{0}^{3}$ band of $\mathrm{C}_{6} \mathrm{H}_{6}$," J. Chem. Phys. (in press).

13. S. Gerstenkorn and P. Luc, Atlas du Spectre d'Absorption de la Molecule de l'Iode (CNRS, Paris, 1978). A correction of $-0.0056 \mathrm{~cm}^{-1}$ was used, according to $\mathrm{S}$. Gerstenkorn and P. Luc, Rev. Phys. Appl. 14, 791 (1979).

14. P. Esherick, A. Owyoung, and J. Pliva, J. Chem. Phys. 83, 3311 (1985).

15. J. H. Callomon, T. M. Dunn, and I. M. Mills, Phil. Trans. R. Soc. London Ser. A 259, 499 (1966).
16. T. A. Stephenson, P. L. Radloff, and S. A. Rice, J. Chem. Phys. 81, 1060 (1984).

17. A. R. Hoy and I. M. Mills, J. Mol. Spectrosc. 46, 333 (1973).

18. A. E. W. Knight, C. S. Parmenter, and M. W. Schuyler, J. Am. Chem. Soc. 97, 1993 (1975).

19. J. T. Hougen, J. Chem. Phys. 37, 1433 (1962).

20. G. Herzberg, Molecular Spectra and Molecular Structure (Van Nostrand Reinhold, New York, 1966), Vol. III, p. 232.

21. Throughout this paper any change $\Delta$ of a given quantity refers to the difference between the value in the electronically excited state $\left(S_{1}\right)$ minus its value in the electronic ground state $\left(S_{0}\right)$. This definition applies to both the excitation (pump) and the dump transition.

22. M. Dang-Nhu and J. Pliva, J. Mol. Spectrosc. 138, 423 (1989).

23. J. Pliva and A. S. Pine, J. Mol. Spectrosc. 93, 209 (1982).

24. A. B. Hollinger and H. L. Welsh, Can. J. Phys. 56, 1513 (1978).

25. H. B. Jensen and S. Brodersen, J. Raman Spectrosc. 8, 103 (1979).

26. B. T. Darling and D. M. Dennison, Phys. Rev. 57, 128 (1940),

27. M. R. Aliev and J. K. G. Watson, in Molecular Spectroscopy: Modern Research, Vol. III, K. N. Rao, ed. (Academic, Orlando, $1985)$, p. 36.

28. S. Brodersen and A. Langseth, Math. Fys. Skr. Dan. Vid. Selsk. 1, 1 (1956).

29. D. A. Chernoff, J. D. Myers, and J. G. Pruett, J. Chem. Phys. 85, 3732 (1986).

30. U. Schubert, E. Riedle, and H. J. Neusser, J. Chem. Phys. 90, 5994 (1989).

31. T. Suzuki and M. Ito, J. Chem. Phys. 91, 4564 (1989).

32. R. H. Page, Y. R. Shen, and Y. T. Lee, J. Chem. Phys. 88, 4621 (1988). 